\title{
On the Complex and Chaotic Dynamics of Standard Logistic Sine Square Map
}

\author{
Sudesh Kumari, Renu Chugh and Radu Miculescu
}

\begin{abstract}
In this article, we set up a new nonlinear dynamical system which is derived by combining logistic map and sine square map in Mann orbit (a two step feedback process) for ameliorating the stability performance of chaotic system and name it Standard Logistic Sine Square Map (SLSSM). The purpose of this paper is to study the whole dynamical behavior of the proposed map (SLSSM) through various introduced aspects consisting fixed point and stability analysis, time series representation, bifurcation diagram and Lyapunov exponent. Moreover, we show that our map is significantly superior than existing other one dimensional maps. We investigate that the chaotic and complex behavior of SLSSM can be controlled by selecting control parameters carefully. Also, the range of convergence and stability can be made to increase drastically. This new system (SLSSM) might be used to achieve better results in cryptography and to study chaos synchronization.
\end{abstract}

\section{Introduction}

In nature, chaos is a nonlinear phenomena of nonlinear dynamical systems which is unpredictable, aperiodic and extreme sensitive on initial values [1,2]. There is diversity of fields in which chaotic maps and fractals are widely studied $[3,4,5,6,7,8,9,10]$.

Key Words: Standard logistic sine square map, periodic and fixed points, chaos, time series plot, bifurcation plot, Lyapunov exponent (LE).

2010 Mathematics Subject Classification: Primary 34H10, 37F45, 37B25. Secondary $37 \mathrm{M} 10,37 \mathrm{C} 25$.

Received: 08.01.2021

Accepted: 19.02 .2021 
The logistic map (a quadratic polynomial which was investigated as a population growth model) is a nonlinear dynamical system, originally due to P.F. Verhulst in 1945 and 1947 [11]. The logistic map is given by the equation

$$
x_{n+1}=\mu x_{n}\left(1-x_{n}\right), n=0,1,2, \ldots,
$$

where $x_{n} \in[0,1]$. It represents the population at any time $n$, where $\mu$ is a control parameter indicating the rate of population growth [13]. May [14] was the first mathematician who published his article on the dynamics of logistic map and it opened a new door for researchers in the field of modern chaos theory due to its simplest form. Nowadays, logistic map has become a milestone and widely applied in every branch of science, for example, in cryptography $[15,16,17,18]$, to control traffic and traffic signals $[25,19,20$, $21,22,23,24]$, to generate chaotic signals for securing communication system [26], as a chaotic noise analog generator and in circuits [27, 28] and in many other fields [29].

Sine map is one dimensional chaotic dynamical system defined in [11] and given by the equation

$$
x_{n+1}=\lambda \sin \left(\pi x_{n}\right), n=0,1,2, \ldots,
$$

where $x_{n} \in[0,1]$ and $\lambda>0$. The qualitative dynamics of the Logistic and sine maps are identical as both are unimodel. Sine map had been used to secure Communication [32], Chaos-based Image Cryptography [33], transmission [34] etc. For more details and applications of sine map one may refer to $[35,36,37$, 38]. In 1993, Xie et al. [30] developed symbolic dynamics for the sine square map, which is related to a hybrid optical bi-stability device and given by

$$
x_{n+1}=\lambda \sin ^{2}\left(x_{n}-B\right),\left|x_{n}-B\right| \leq \pi, n=0,1,2 \ldots .
$$

Philominathan et al. [31] studied the statistical dynamics of sine square map.

In 2nd decade of 21st century, researchers (Wu et al. [35], de Carvalho et al. [36], Wu et al. [37], Hua et al. [38] and many others) studied the chaotic behavior of a dynamical system which is obtained by connecting two dynamical systems. They demonstrated that obtained new systems have excellent ergodicity, extreme improved chaotic range and more complex chaotic behavior than existing systems. These features encouraged authors to apply new systems to get better outputs in cryptography, signal processing, bioengineering, and secure communication systems. These facts inspired us to investigate a new dynamical system by connecting two dynamical systems.

Mann [39] investigated a fixed point iterative method. Authors [40] used this method to study the dynamical properties of the logistic map. They found that by applying this method the range of convergence and stability of 
the logistic map increased drastically to a larger value of control parameters. Recently, Ashish et al. [22, 41] studied the logistic map in superior orbit and they presented a better Traffic control model.

In this article, we set up a new dynamical system which is obtained by connecting logistic map and sine square map in superior orbit and call it Standard Logistic Sine Square Map (SLSSM). The whole paper is divided into four sections. A brief introduction is given in Section 1. In Section 2, some basic definitions are recalled that have been taken into account during our study. Section 3 is dedicated to the formation of a new dynamical system (SLSSM). This section is further divided into five subsections in which entire dynamical behavior of SLSSM has been detected by adopting fixed point and stability analysis, time series representation, bifurcation diagram and Lyapunov exponent techniques. In Section 4, we prove the superiority of SLSSM by comparing its stability and chaos performance with existing other one dimensional maps. Finally, we present a brief discussion and concluding remarks in Section 5.

\section{Preliminaries}

This section deals with basic definitions, facts and notations which are roots of our study.

Definition 2.1. [11]. Let $X$ be a nonempty subset of real numbers and $g$ be a self-map on $X$, i.e., $g: X \rightarrow X$. For a point $x_{0} \in X$, the Picard Orbit $(P O)$ is the set of all the iterates of a point $x_{0}$, i.e.,

$$
P O\left(g, x_{0}\right)=\left\{x_{n}: x_{n}=g\left(x_{n-1}\right), n=1,2, \ldots\right\} .
$$

Here, the orbit of the initial point $x_{0}$ (also known as seed) represents a sequence $g^{n}\left(x_{0}\right)$ with respect to $g$.

Definition 2.2. (Fixed point). Consider a mapping $g: X \rightarrow X$, where $X$ is a nonempty set. Then, the point $x_{0} \in X$ is known as fixed point if $g\left(x_{0}\right)=$ $x_{0}[11]$.

Definition 2.3. (Periodic point). Let $X$ be a nonempty subset of real numbers and $g: X \rightarrow X$ be a mapping. A point $x_{0} \in X$ is said to be periodic point of $g$ with period $q$ if $g^{q}\left(x_{0}\right)=x_{0}$. The point $x_{0}$ has prime period $m$ if $g^{m}\left(x_{0}\right)=x_{0}$ and $g^{n}\left(x_{0}\right) \neq x_{0}$ for $0<n<m$ [11].

The orbit $P O\left(g, x_{0}\right)$ is said to be periodic orbit or periodic cycle if the point $x_{0}$ is a periodic point.

Definition 2.4. [11]. Let $g: \mathbb{R} \rightarrow \mathbb{R}$ be a differentiable map having a fixed point $x_{0}$. Then, the fixed point $x_{0}$ is known as: 
1. attracting (stable) fixed point or sink if $\left|g^{\prime}\left(x_{0}\right)\right|<1$,

2. repelling (unstable) fixed point or source if $\left|g^{\prime}\left(x_{0}\right)\right|>1$,

3. neutral (indifferent) if $\left|g^{\prime}\left(x_{0}\right)\right|=1$, where $g^{\prime}\left(x_{0}\right)$ is the first order derivative of the map $g$ at $x=x_{0}$.

Definition 2.5. (Lyapunov exponent). Consider a differentiable map $g: \mathbb{R} \rightarrow$ $\mathbb{R}$. The Lyapunov exponent (LE) of $g$ for an orbit $\left\{a_{n}\right\}$ is defined by

$$
\sigma\left(a_{1}\right)=\lim _{n \rightarrow \infty} \frac{1}{n} \sum_{i=1}^{n} \ln \left|g^{\prime}\left(a_{i}\right)\right|,
$$

provided this limit exists [12].

Definition 2.6. Let $g: X \rightarrow X$ be a map, where $X$ is a nonempty set. Consider a sequence $\left\{z_{n}\right\}$ of iterates for initial point $z_{0} \in X$ in the following way

$$
z_{n+1}=\alpha_{n} g\left(z_{n}\right)+\left(1-\alpha_{n}\right) z_{n}, n=1,2,3, \ldots,
$$

where $\alpha_{n}$ is a sequence of positive numbers in $[0,1]$ for each $n$. Then, this sequence is called Superior orbit [40], which is originally due to W.R. Mann [39]. If we take $\alpha_{n}=1$ then, Superior orbit (2.3) reduces in Picard orbit (2.1).

\section{Formation of the Dynamical System SLSSM}

We construct a dynamical system i.e., Standard Logistic Sine Square Map (SLSSM) which is established by combining two dynamical systems, namely, logistic map and sine square map in superior orbit (2.3). The proposed map $S_{\mu, \alpha_{n}}$ is given by

$S_{\mu, \alpha_{n}}\left(x_{n}\right)=x_{n+1}=\alpha_{n} \mu x_{n}\left(1-x_{n}\right)+\alpha_{n} \lambda \sin ^{2}\left(2 \pi x_{n}\right)+\left(1-\alpha_{n}\right) x_{n}, n=0,1,2, \ldots$,

where $x_{n}=x \in[0,1]$ and $\alpha_{n} \in[0,1]$ is a sequence. For simplicity, we take $\alpha_{n}=\alpha \in[0,1]$ throughout this article. Also, parameters $\alpha, \mu, \lambda \in[0,1]$ alter the dynamical behavior of SLSSM and control the intensity level of nonlinear perturbed map given by (3.1), i.e., $\alpha, \mu$ and $\lambda$ are known as control parameters. We adopt the following experimental methodologies to analyze the whole dynamical behavior of the system SLSSM by running a program in Matlab. 


\subsection{Fixed point and stability analysis of SLSSM}

The fixed points of the map (3.1) can be found out by using Definition 2.2, therefore, to find fixed points, we can write

$$
\begin{array}{r}
S_{\mu, \alpha}(x)=x \\
\text { i.e., } \alpha \mu x(1-x)+\alpha \lambda \sin ^{2}(2 \pi x)+(1-\alpha) x=x \\
\alpha \mu x(1-x)+\alpha \lambda \sin ^{2}(2 \pi x)-\alpha x=0
\end{array}
$$

The map $S_{\mu, \alpha}(x)$ has two fixed points being a quadratic equation. One can instantly observe that $x=0$ is always a fixed point of $S_{\mu, \alpha}(x)$. It is difficult to calculate analytically second fixed point due to non-linearity of the system. The control parameters affect it, i.e., second fixed point depends on parameters $\alpha, \mu$ and $\lambda$. The map $S_{\mu, \alpha}$ is iterated 100 times, i.e., 100 number of iterations have been observed to compute the fixed points (see Table 1) for all $x \in[0,1]$, in which one parameter, say $\lambda=0.2$ (some other values can be taken) has been considered as fixed. Moreover, the maximum value of parameter $\mu$ has also been obtained for which the system SLSSM remains convergent and stable. Here, we compute fixed points upto four decimal points by taking the maximum value of $\mu$ upto two decimal points for which the system SLSSM remains convergent .

\begin{tabular}{|l|l|l|l|}
\hline \multirow{2}{*}{$\alpha$} & \multirow{2}{*}{ Fixed Point } & \multicolumn{2}{|c|}{ Maximum value of $\mu$} \\
\cline { 3 - 4 } & & for convergence & for stability \\
\hline 0.9 & 0.7381 & 2.79 & 3.99 \\
\hline 0.8 & 0.7474 & 2.90 & 4.38 \\
\hline 0.7 & 0.7548 & 3.00 & 4.47 \\
\hline 0.6 & 0.7752 & 3.33 & 4.82 \\
\hline 0.5 & 0.7938 & 3.72 & 5.31 \\
\hline 0.4 & 0.8191 & 4.42 & 6.00 \\
\hline 0.3 & 0.8550 & 5.89 & 7.23 \\
\hline 0.2 & 0.9005 & 9.29 & 9.70 \\
\hline 0.1 & 0.9419 & 16.75 & 16.75 \\
\hline
\end{tabular}

Table 1: Fixed points and range of convergence and stability of SLSSM

From Table 1, we observe that whole dynamical behavior of SLSSM depends on parameter $\alpha$. As we decrease the value of $\alpha$, system remains stable for a larger value of $\mu$. Thus, the system can be stabilized for larger values of $\mu$ by reducing the value of parameter $\alpha$, i.e., the range of convergence and stability of SLSSM can be increased drastically upto $\mu=16.75$. 
Further, fixed points exist where the diagonal line $y=x$ intersects the map $S_{\alpha, \mu}$ as shown in Fig. 1 by $a$ and $b$. We have presented the fixed points $a$ and $b$ at $\alpha=0.9$ for $\mu=2.79$.

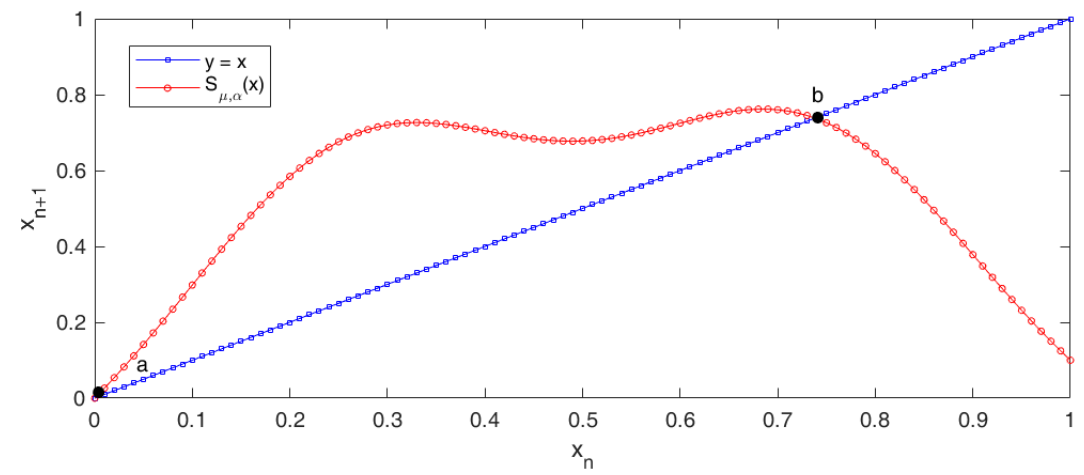

Fig. 1. Graphical representation of fixed points of $S_{\mu, \alpha}(x)$ at $\alpha=0.9$ for $\mu=2.79$

\subsection{Time series representation of SLSSM for $\alpha=0.9,0.5$ and 0.1}

In this section, adopting time series depiction of SLSSM, we attempt to corroborate the fixed points and stability results given in Table 1 experimentally. We obtain the optimum value of $\mu$ for different choices of $\alpha$ against some initial values of $x \in[0,1]$ using 100 number of iterations.

Example 3.1. Describe the whole dynamical behavior of SLSSM (3.1) for $\alpha=0.9$ and for all $x \in[0,1]$ by using time series representation of dynamical systems.

Solution. We analyze the whole dynamical behavior of SLSSM for $\alpha=0.9$ by portraying Figs. 2, 3, 4, 5 and 6 . We observe from Fig. 2 that the trajectory of SLSSM converges to a fixed point for $\mu \leq 2.79$ for all values of $x$. The system SLSSM exhibits more complicated behavior for $2.79<\mu \leq 3.87$, as system fluctuates between 2, 4 and 8 stable fixed points. The orbit oscillates between 2 stable fixed points for all $x \in[0,1]$ and for $\mu \in(2.79,3.09] \cup[3.68,3.82]$ as depicted in Fig. 3 at $\mu=3.82$. There exists 4 stable solutions of the system or orbit vibrates between 4 stable fixed points for $\mu \in(3.10,3.23] \cup[3.37,3.49] \cup$ $[3.58,3.67] \cup[3.83,3.86]$. We have illustrated this fact by Fig. 4 for $\mu=3.20$. Furthermore, the fluctuation in the orbit tends to 8 stable fixed points when the parameter $\mu$ lies in the range [3.24,3.36] $\cup[3.50,3.54]$ (see Fig. 5). Also, as we increase $\mu$ from $\mu=3.86$, more and more oscillations take place in the 
orbit of the system. Ultimately, chaos starts to exists, i.e., system becomes chaotic. This behavior is depicted in Fig. 6 for $\mu=3.92$.

Remark 3.2. The proposed dynamical system SLSSM (3.1) is more interesting than that of logistic map (1.1) and sine map (1.3) due to its marvellous and complex periodic behavior. Unlike these systems, SLSSM has the property to exhibit periodic fixed points of periods 4 and 2 even after the occurrence of periodic fixed points of periods 8, i.e., the system possesses the attribute of reducing the periodicity of the periodic fixed points.

Note 3.3. One cannot easily interpret the behavior of SLSSM only by computing starting range of parameter $\mu$. It is requirement of the system that its behavior should be examined for all values of $\mu \in[2.80,3.86]$ as explained in Example 3.1.

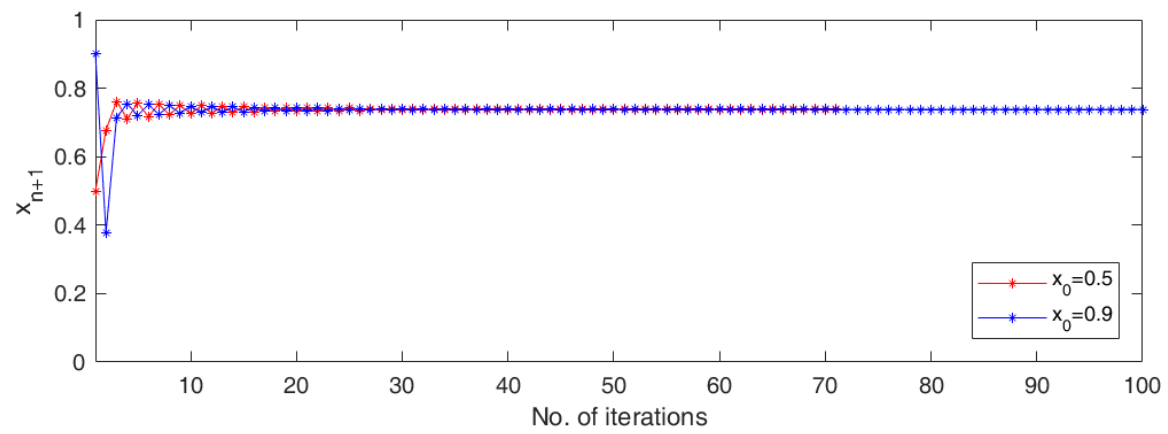

Fig. 2. Stable convergent solution of SLSSM for $\alpha=0.9, \mu=2.79$

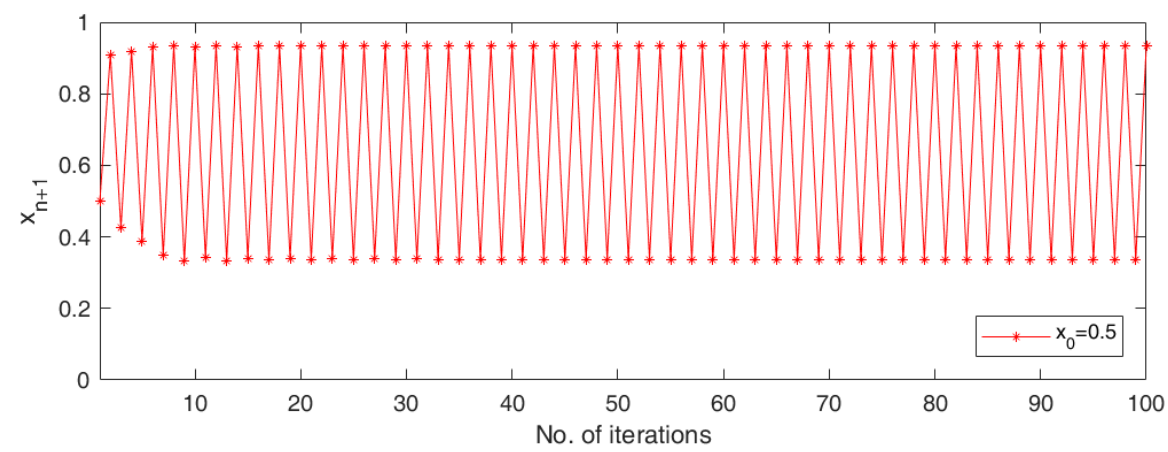

Fig. 3. 2-Stable fixed point oscillation of SLSSM for $\alpha=0.9, \mu=3.82$ 


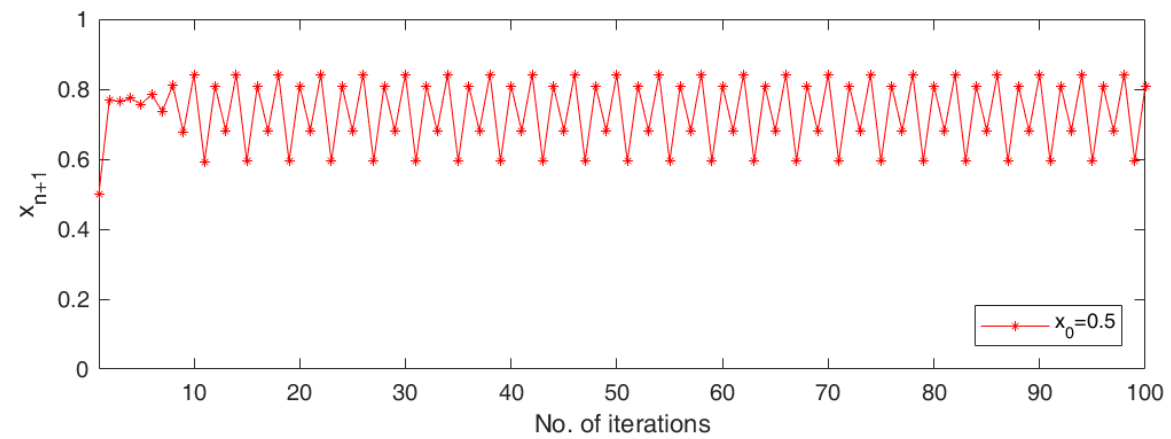

Fig. 4. 4-Stable fixed point oscillation of SLSSM for $\alpha=0.9, \mu=3.20$

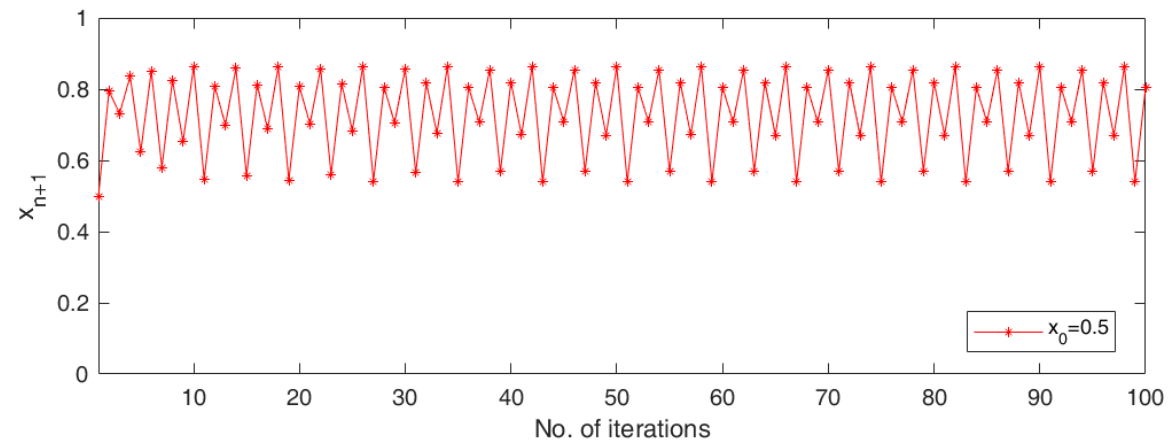

Fig. 5. 8-Stable fixed point oscillation of SLSSM for $\alpha=0.9, \mu=3.31$

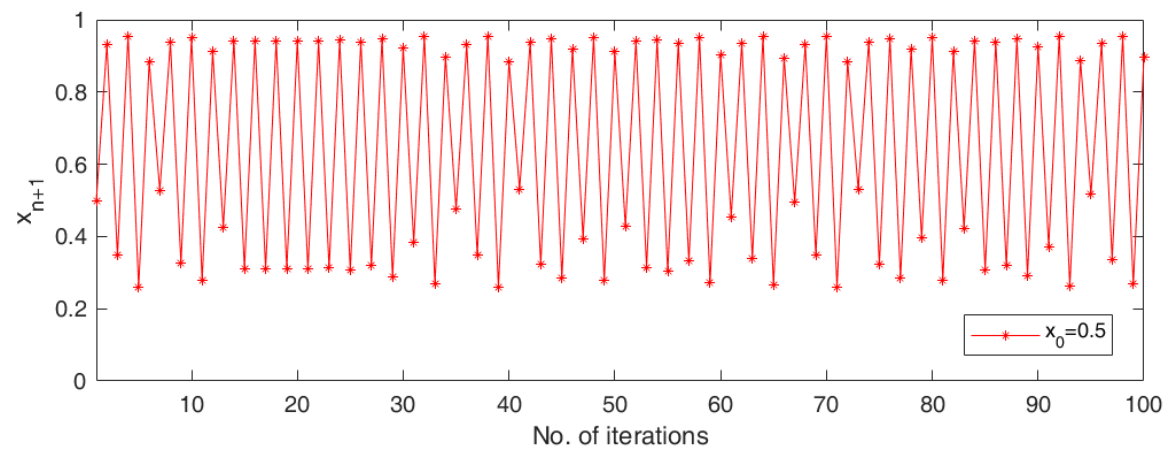

Fig. 6. Divergent behavior of SLSSM for $\alpha=0.9, \mu=3.92$

Example 3.4. By drawing time series diagram, examine the whole dynamical behavior of SLSSM (3.1) for $\alpha=0.5$ and for all $x \in[0,1]$ by taking 100 number 
of iterations.

Solution. For this specific value of parameter $\alpha$, the system has stable fixed point for $0<\mu \leq 3.72$ for all $x \in[0,1]$. This fact is presented in Fig. 7 for $x_{0}=0.5$ and $\mu=3.72$. The trajectory of SLSSM fluctuates between 2 fixed points for $3.72<\mu \leq 4.76$ as shown in Fig. 8 at $\mu=4.75$. Further, orbit starts to show more and more vibrations, i.e., the system exhibits sensitive dependence on initiators when parameter $\mu$ increases through $\mu>5$.4. Fig. 9 shows this chaoticity of SLSSM for $x_{0}=0.9$ at $\mu=6$.

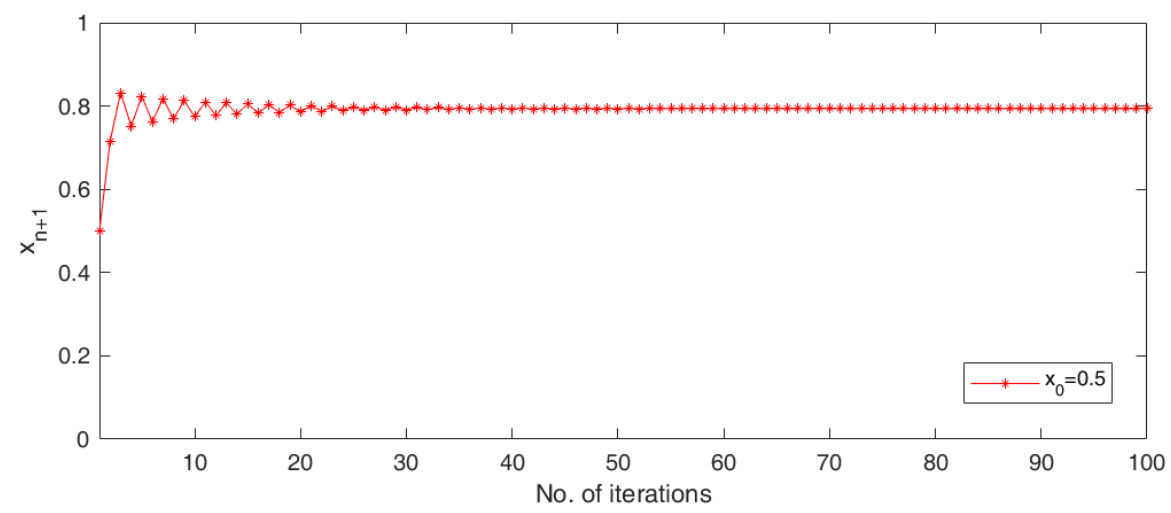

Fig. 7. Stable convergent solution of SLSSM for $\alpha=0.5, \mu=3.72$

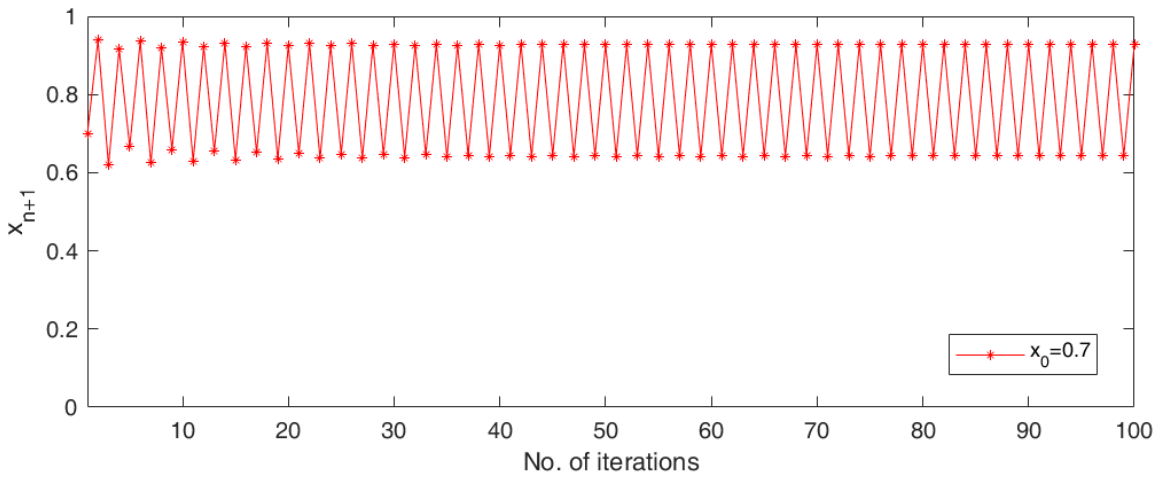

Fig. 8. 2-Stable fixed point fluctuation of SLSSM for $\alpha=0.5, \mu=4.75$ 


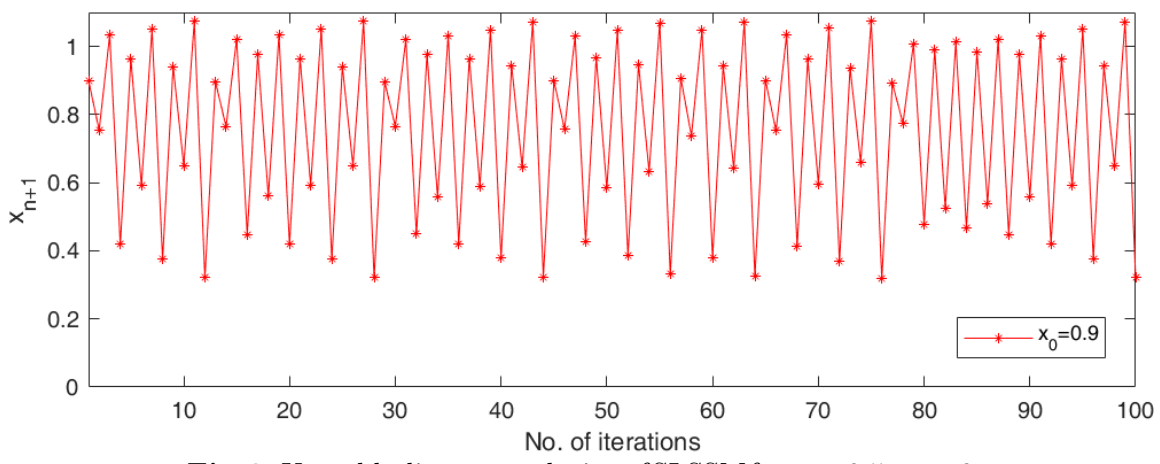

Fig. 9. Unstable divergent solution of SLSSM for $\alpha=0.5, \mu=6$

Example 3.5. Show that the stability of the map $S_{\alpha, \mu}(x)$ can be extended by decreasing the parameter $\alpha$. Explain this fact for all $x \in[0,1]$ by taking $\alpha=0.1$.

Solution. The proposed map $S_{\alpha, \mu}(x)$ given by (3.1), converges to a stable fixed point 0.9419 when the parameter $\mu$ exists in the range $(0,16.75]$. This convergent behavior is depicted in Fig. 10 for all $x_{0} \in[0,1]$ at $\mu=16$. Moreover, the map $S_{\alpha, \mu}(x)$ cannot be defined for all $\mu>16.75$, since in this range $x_{n+1}>1$ as shown in Fig. 11 for $\mu=16.79$ which represents the undefined behavior of the system.

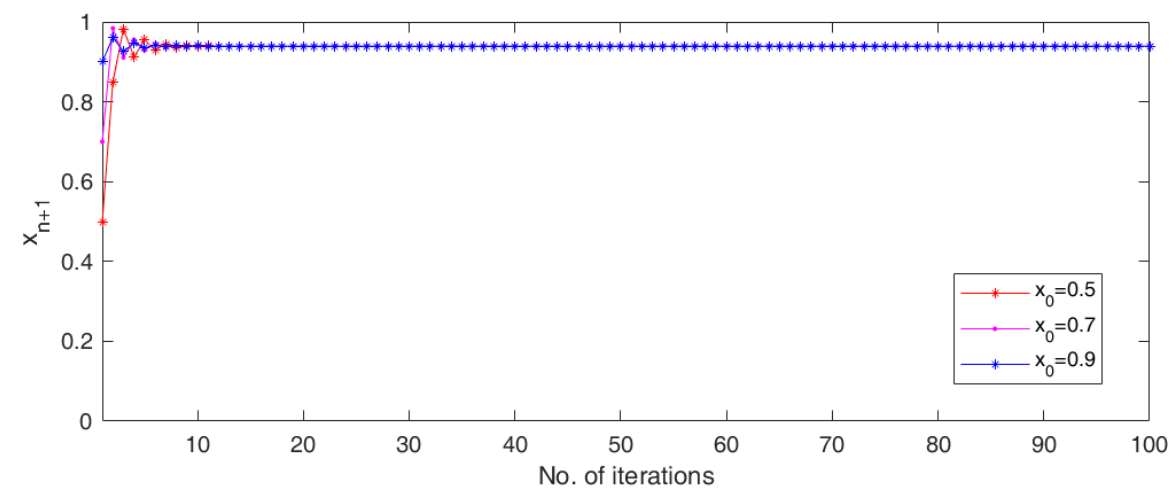

Fig. 10. Stable convergent fixed point of SLSSM for $\alpha=0.1, \mu=16$ 


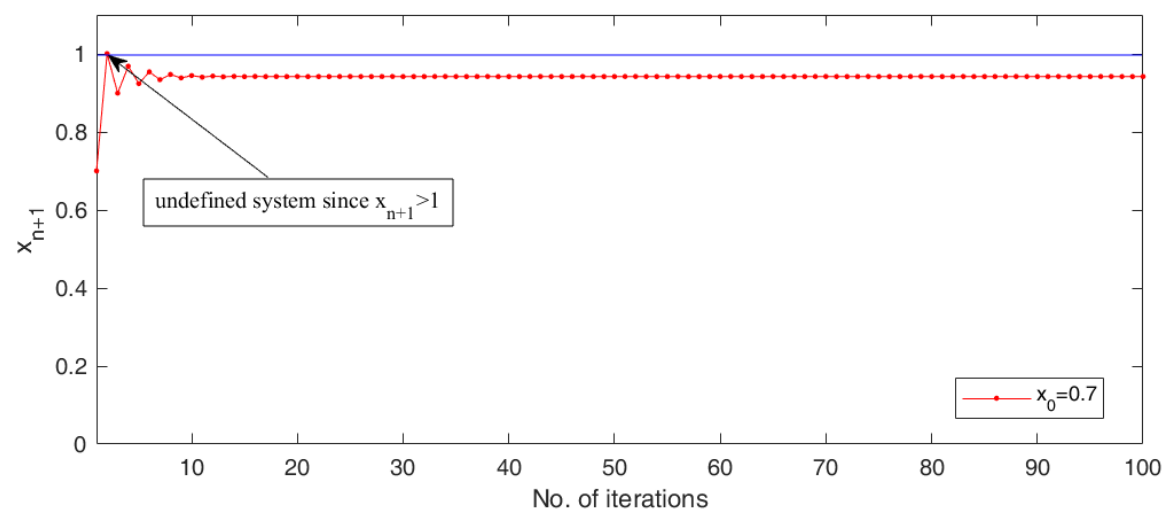

Fig. 11. Undefined SLSSM for $\alpha=0.1, \mu=16.79$

\subsection{Bifurcation analysis of SLSSM for different choices of $\mu$}

The bifurcation diagrams are the tools generally used to classify the dynamical systems in nonlinear regimes. Bifurcation diagrams exhibit an abrupt change which occurs in the asymptotic solutions of a dynamical system.

In this section, the whole dynamical behavior of the system SLSSM (3.1) is presented by drawing bifurcation diagrams for $\alpha=0.9,0.5$ and 0.1 . A route from periodic regime to chaotic regime has been demonstrated in Figs. 12, 13,14 by letting step size of $\mu=0.001$, initiator $x_{0}=0.5$ and the number of iterations $(\mathrm{N})=800$.

In Fig. 12, the whole dynamical system SLSSM has been split into different regimes which interpret the complication of the system. For $0<\mu \leq 2.82$, SLSSM has a stable fixed point and a period doubling bifurcation occurs for $2.82<\mu \leq 3.16$ as depicted by regimes of period 1 and 2. Further, SLSSM represents a route from more than 2 periods to 2 periods for $3.16<\mu \leq 3.67$. The system acquires a route from periodic to chaos when parameter $\mu$ exceeds from 3.86, i.e., for $\mu>3.86$, where the system shows sensitive dependence on initiators. 


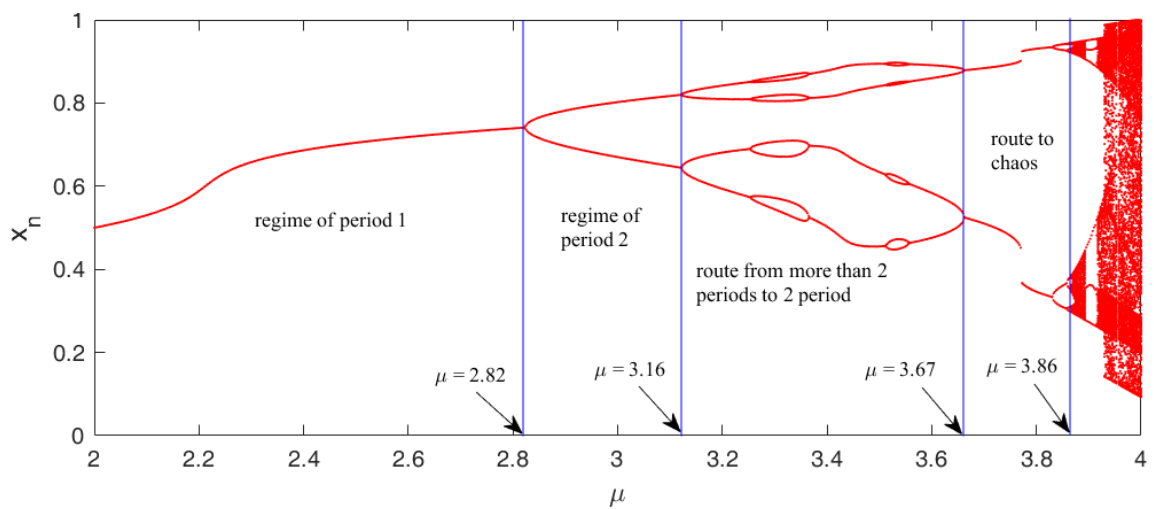

Fig. 12. Bifurcation plot of SLSSM for $2 \leq \mu \leq 4, \alpha=0.9, x_{0}=0.5$

Furthermore, the period doubling bifurcation of SLSSM is also presented for $\alpha=0.5$ by Fig.13. In the figure, SLSSM has stable solutions for $0<\mu \leq$ 5.31. The system cannot be defined when the parameter $\mu$ exceeds from 5.31 as described by undefined regime.

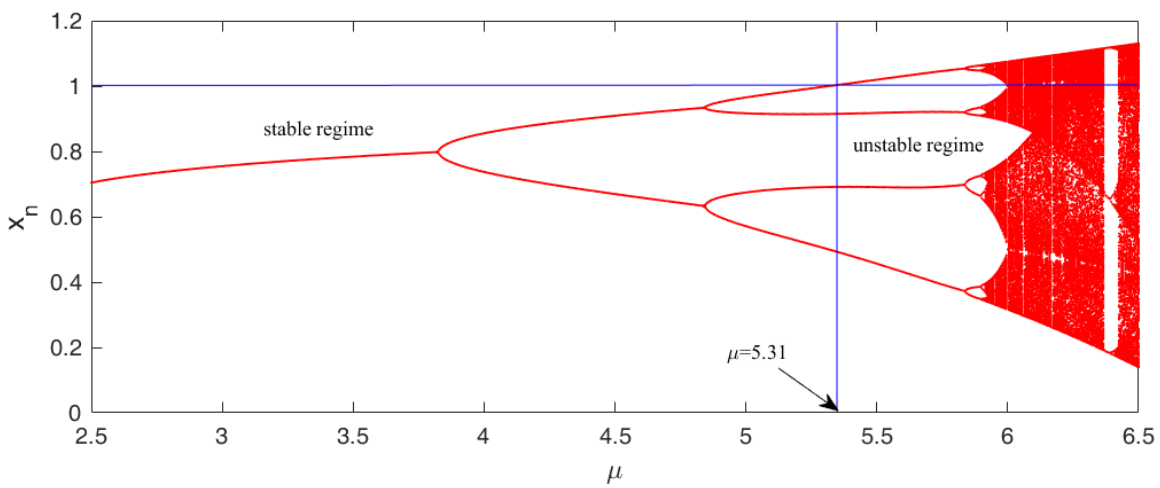

Fig. 13. Bifurcation plot of SLSSM for $2.5 \leq \mu \leq 6.5, \alpha=0.5, x_{0}=0.5$

Moreover, from Fig. 14, we observe that SLSSM remains stable for an extendable range of $\mu$, i.e., for $0<\mu \leq 20.34$ since orbit converges to a fixed point. The system cannot be defined if the value of $\mu$ increases through $\mu=20.34$ since in this range orbit $x_{n}>1$, i.e., $x_{n} \notin[0,1]$ which demonstrates the undefined behavior of the system SLSSM. 


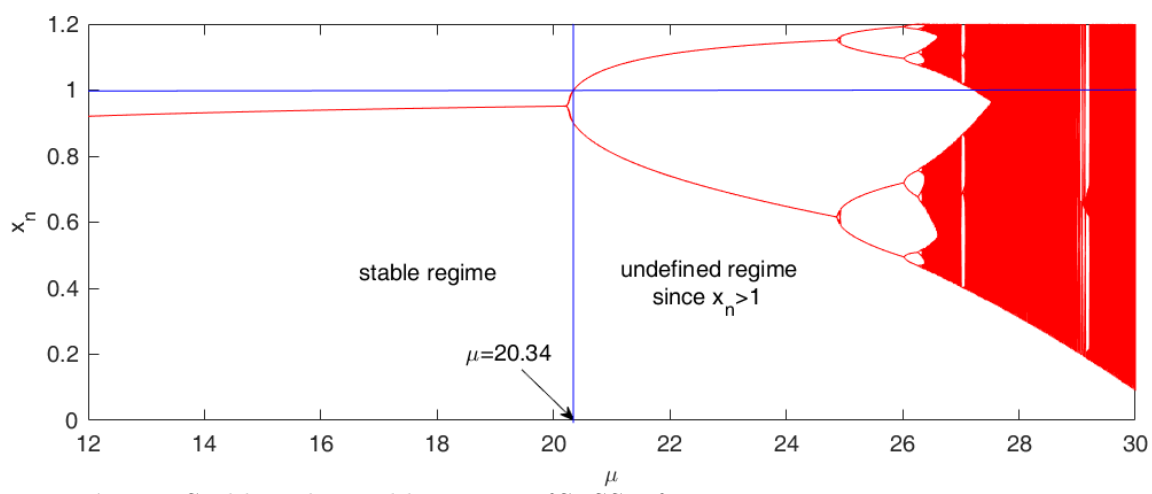

Fig. 14. Stable and unstable regimes of SLSSM for $12 \leq \mu \leq 30, \alpha=0.1, x_{0}=0.5$

Remark 3.6. The system SLSSM acquires more and more dynamical properties when the value of parameter $\alpha \in[0,1]$ increases as described by bifurcation diagrams, i.e., for $\alpha=0.1,0.5$, the system exhibits fixed point and periodic properties; for $\alpha=0.9$, system has fixed point, periodicity, reduction in periodicity and chaotic properties.

\subsection{Mathematical and experimental approach of SLSSM by Lya- punov exponent}

The Lyapunov exponent (LE) enables one to measure the chaoticity of a nonlinear dynamical system. It measures the degree at which orbits starting from very close initial points diverge or converge over time. If the orbits diverse exponentially, the system shows sensitive dependence on initial conditions, i.e., the system becomes chaotic. Further, the system becomes dissipative (stable) if the orbits converge. We are going to estimate the Lyapunov exponent only for finite orbits as considering infinite orbits is impossible. The Lyapunov exponent for the dynamical system SLSSM is described in the following manner:

We start by considering two nearby initial orbits $S_{\mu, \alpha}^{n}(x)$ and $S_{\mu, \alpha}^{n}(x+h)$, where $0<h<1, \alpha \in(0,1), \mu>0$ and $n$ denotes the number of iterations. Let us express the divergence $\Delta$ of these orbits by an exponential growth $h e^{\sigma n}$. Then, we have

$$
\begin{aligned}
& S_{\mu, \alpha}^{n}(x+h)-S_{\mu, \alpha}^{n}(x)=\Delta, \\
& \text { i.e., } S_{\mu, \alpha}^{n}(x+h)-S_{\mu, \alpha}^{n}(x)=h e^{\sigma n} \text {. }
\end{aligned}
$$

This implies

$$
\frac{S_{\mu, \alpha}^{n}(x+h)-S_{\mu, \alpha}^{n}(x)}{h}=e^{\sigma n} .
$$


Letting limit as $h \rightarrow 0$ on each side of (3.2), we have

$$
\lim _{h \rightarrow 0} \frac{S_{\mu, \alpha}^{n}(x+h)-S_{\mu, \alpha}^{n}(x)}{h}=\lim _{h \rightarrow 0} e^{\sigma n} .
$$

This gives

$$
\left(S_{\mu, \alpha}^{n}\right)^{\prime}(x)=e^{\sigma n}
$$

Taking logarithm on both side, we have

$$
\sigma=\frac{1}{n} \ln \left|\left(S_{\mu, \alpha}^{n}\right)^{\prime}(x)\right|
$$

where $\left(S_{\mu, \alpha}^{n}\right)^{\prime}(x)$ represents the first order derivative of $S_{\mu, \alpha}^{n}(x)$. The map $S_{\mu, \alpha}^{n}(x)$ is a recursive map, therefore, we have $x_{2}=S_{\mu, \alpha}\left(x_{1}\right), x_{3}=S_{\mu, \alpha}\left(x_{2}\right), x_{4}=$ $S_{\mu, \alpha}\left(x_{3}\right), \ldots, x_{n+1}=S_{\mu, \alpha}\left(x_{n}\right)$, then, using chain rule of derivatives, $\left(S_{\mu, \alpha}^{n}\right)^{\prime}\left(x_{1}\right)$ can be written as

$$
\left(S_{\mu, \alpha}^{n}\right)^{\prime}\left(x_{1}\right)=S_{\mu, \alpha}^{\prime}\left(x_{n}\right) \cdot S_{\mu, \alpha}^{\prime}\left(x_{n-1}\right) \ldots S_{\mu, \alpha}^{\prime}\left(x_{2}\right) \cdot S_{\mu, \alpha}^{\prime}\left(x_{1}\right)
$$

Using (3.5), (3.4) reduces to

$$
\sigma=\frac{1}{n}\left\{\ln \left|S_{\mu, \alpha}^{\prime}\left(x_{n}\right) \cdot S_{\mu, \alpha}^{\prime}\left(x_{n-1}\right) \ldots S_{\mu, \alpha}^{\prime}\left(x_{2}\right) \cdot S_{\mu, \alpha}^{\prime}\left(x_{1}\right)\right|\right\} .
$$

Now, applying the property of logarithm, the value of $\sigma$ can be expressed as

$$
\begin{gathered}
\sigma=\frac{1}{n}\left\{\ln \left|S_{\mu, \alpha}^{\prime}\left(x_{n}\right)\right|+\ln \left|S_{\mu, \alpha}^{\prime}\left(x_{n-1}\right)\right|+\ldots+\ln \left|S_{\mu, \alpha}^{\prime}\left(x_{2}\right)\right|+\ln \left|S_{\mu, \alpha}^{\prime}\left(x_{1}\right)\right|\right\} \\
\sigma=\frac{1}{n} \sum_{i=1}^{n} \ln \left|S_{\mu, \alpha}^{\prime}\left(x_{i}\right)\right|
\end{gathered}
$$

which is required Lyapunov exponent of SLSSM.

From this definition, we draw the following results about the behavior of the dynamical system SLSSM in the form of remarks.

Remark 3.7. The above discussion of Lyapunov exponent $\sigma$, can be used to predict how much the dynamical system SLSSM depends on initial conditions. For $\sigma>0$, the system exhibits extreme sensitive dependence on initial conditions and for $\sigma<0$, the system remains dissipative (stable).

Remark 3.8. For periodic orbit with any period $q, \sigma$ takes the form

$$
\sigma=\frac{1}{q} \sum_{i=1}^{q} \ln \left|S_{\mu, \alpha}^{\prime}\left(x_{i}\right)\right|
$$


Remark 3.9. Particularly, for $q=1$, i.e., for fixed orbit, Lyapunov exponent $\sigma$ reduces to

$$
\sigma=\ln \left|S_{\mu, \alpha}^{\prime}\left(x_{1}\right)\right| .
$$

Example 3.10. Evaluate the dynamical behavior of the map $S_{\mu, \alpha}(x)=$ $\alpha \mu x(1-x)+\alpha \lambda \sin ^{2}(2 \pi x)+(1-\alpha) x ; x \in[0,1]$ by plotting Lyapunov exponent at $\alpha=0.9$ for $1 \leq \mu \leq 4.5$. Further, examine the behavior of fixed and periodic orbits by computing Lyapunov exponent for

(a) $\mu=2.5, \alpha=0.9$ and

(b) $\mu=3.4, \alpha=0.9$.

Solution. (a) We have shown in Section 3.3, that each orbit of SLSSM is a fixed orbit for $0<\mu \leq 2.82$ and $x \in[0,1]$. The fixed point is 0.7045 for $\mu=2.5$. The Lyapunov exponent $(\sigma)$ is determined by solving (3.8). Here,

$$
S_{\mu, \alpha}(x)=\alpha \mu x(1-x)+\alpha \lambda \sin ^{2}(2 \pi x)+(1-\alpha) x,
$$

and

$$
\left(S_{\mu, \alpha}\right)^{\prime}(x)=\alpha \mu(1-2 x)+2 \pi \alpha \lambda \sin (4 \pi x)+(1-\alpha) .
$$

Putting $\alpha=0.9, \mu=2.5$ and $x=0.7045$, we obtain

$$
\begin{aligned}
\left(S_{2.5,0.9}\right)^{\prime}(0.7045)= & 0.9 \times 2.5(1-2 \times 0.7045)+2 \pi \times 0.9 \times 0.2 \sin (4 \pi \times 0.7045) \\
& +(1-0.9)=-0.6462 \text { radian }
\end{aligned}
$$

Next, substituting the value of $\left(S_{2.5,0.9}\right)^{\prime}(0.7045)$ in (3.8), we have

$$
\sigma=\ln |-0.6462|=-0.4366 \text {. }
$$

Thus, the estimated value of Lyapunov exponent is -0.4366 , i.e., $\sigma<0$, therefore, orbit of $x=0.7045$ is stable. The fixed point 0.7045 becomes the stable attractor for the map $S_{2.5,0.9}(0.7045)$.

(b) We observe from Section 3.3, that for $\mu=3.4$, and for all $x \in[0,1]$, the orbit of SLSSM is periodic of period-4. Thus, the periodic points are evaluated as $x_{1}=0.8152, x_{2}=0.6937, x_{3}=0.8779$ and $x_{4}=0.5024$ for $\mu=3.4$. The Lyapunov exponent $(\sigma)$ is determined by solving (3.7) for $q=4$. From (3.9), we have

$$
\begin{aligned}
\left(S_{3.4,0.9}\right)^{\prime}\left(x_{1}\right) & =0.9 \times 3.4(1-2 \times 0.8152)+2 \pi \times 0.9 \times 0.2 \sin (4 \pi \times 0.8152)+(1-0.9) \\
& =-2.6554 \text { radian } \\
\left(S_{3.4,0.9}\right)^{\prime}\left(x_{2}\right) & =0.9 \times 3.4(1-2 \times 0.6937)+2 \pi \times 0.9 \times 0.2 \sin (4 \pi \times 0.6937)+(1-0.9)
\end{aligned}
$$




$$
\begin{aligned}
& =-0.3504 \text { radian } \\
\left(S_{3.4,0.9}\right)^{\prime}\left(x_{3}\right) & =0.9 \times 3.4(1-2 \times 0.8779)+2 \pi \times 0.9 \times 0.2 \sin (4 \pi \times 0.8779)+(1-0.9) \\
& =-3.3429 \text { radian } \\
\left(S_{3.4,0.9}\right)^{\prime}\left(x_{4}\right) & =0.9 \times 3.4(1-2 \times 0.5024)+2 \pi \times 0.9 \times 0.2 \sin (4 \pi \times 0.5024)+(1-0.9) \\
& =0.1194 \text { radian }
\end{aligned}
$$

To determine the value of $\sigma$, we use (3.10), (3.11), (3.12) and (3.13) in (3.7), we have

$$
\begin{aligned}
\sigma & =\frac{1}{4}\{\ln |-2.6554|+\ln |-0.3504|+\ln |-3.3429|+\ln |0.1194|\} \\
& =\frac{1}{4}\{0.4241-0.4554+0.5241-0.9230\} \\
& =\frac{1}{4}(-0.4302) \\
\text { i.e., } \sigma & =-0.1076
\end{aligned}
$$

Thus, the determined value of Lyapunov exponent is -0.1076 , which is negative. Therefore, periodic points $x_{1}, x_{2}, x_{3}$ and $x_{4}$ are the stable attractors for the map $S_{\mu, \alpha}(x)$.

In Fig. 15, we plot Lyapunov exponent $(\sigma)$ to ascertain the behavior of dynamical system $S_{\mu, \alpha}(x)$ for $1 \leq \mu \leq 4.5$ at $\alpha=0.9$. To plot it, we consider 10, 000 iterations i.e. $N=10,000$ and initiator $x_{0}=0.5$. From the figure, it is clear that SLSSM has remained stable for $0<\mu \leq 3.86$ since in this range $\sigma<0$, i.e., the system possesses stable orbits. Also, orbit in the zoomed rectangular area, shows extreme sensitive dependence on initial condition as here $\sigma>0$. Thus, chaos may be occurred in the system when we increase the parameter $\mu$ through $\mu=3.86$. 


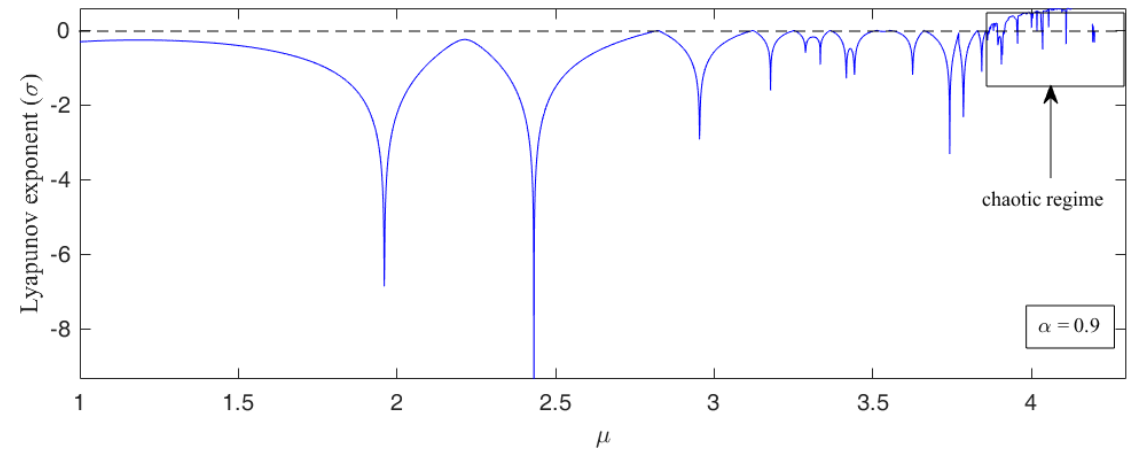

Fig. 15. Lyapunov exponent diagram of SLSSM for $x_{0}=0.5,1 \leq \mu \leq 4.5$ at $\alpha=0.9$

Example 3.11. Explain the dynamical behavior of the map $S_{\mu, \alpha}(x)=\alpha \mu x(1-$ $x)+\alpha \lambda \sin ^{2}(2 \pi x)+(1-\alpha) x ; x \in[0,1]$ by plotting Lyapunov exponent for the following values of parameters $\mu$ and $\alpha$ :

(a) $1 \leq \mu \leq 7$ at $\alpha=0.7$,

(b) $1 \leq \mu \leq 30$ at $\alpha=0.1$.

Solution. (a) We determine the dynamical behavior of SLSSM by drawing the Lyapunov exponent diagram (see Fig. 16) for the given values of parameters and initiator $x_{0}=0.5$. We notice that for $0<\mu \leq 5.9$, the maximum Lyapunov exponent $\sigma$ remains negative, which expresses the stable behavior of the system. For the increased range of parameter $\mu$, i.e., for $5.9<\mu \leq 7$, the spectrum of Lyapunov exponents starts to approach to a positive value of $\sigma$, which is an indication of the chaos in the dynamical system SLSSM.

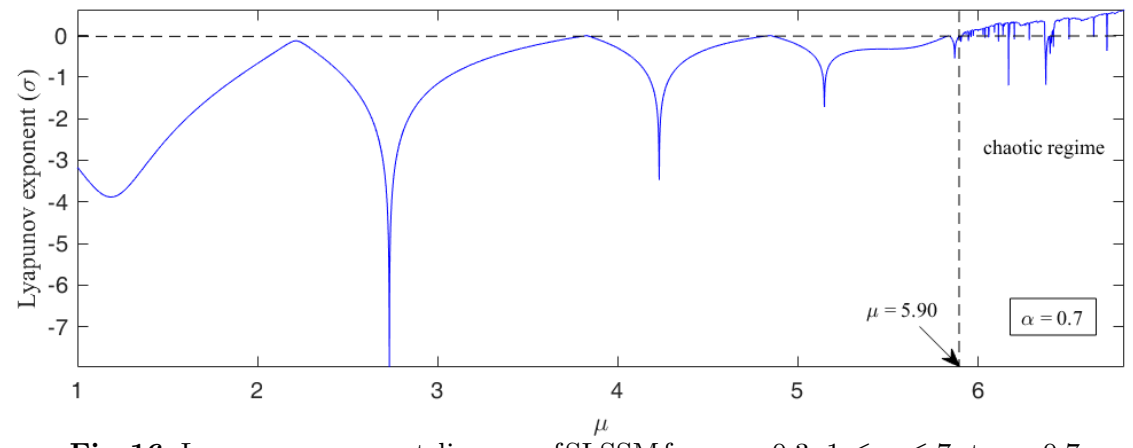

Fig. 16. Lyapunov exponent diagram of SLSSM for $x_{0}=0.3,1 \leq \mu \leq 7$ at $\alpha=0.7$

Solution. (b) The stability of dynamical systems can be increased by controlling the parameters, we analyze this fact by estimating the value of Lya- 
punov exponent $\sigma$ at a decreased value of parameter $\alpha$, i.e., at $\alpha=0.1$. At this particular value of $\alpha$, the system SLSSM exhibits stable behavior for an increased range of parameter $\mu$, i.e., for $0<\mu \leq 20.34$ whose all orbits converge to a fixed point.

Now, we determine the Lyapunov exponent $(\sigma)$ for $\alpha=0.1, \mu=20$ and $x=0.9510$ by solving $(3.8)$.

From (3.9), we obtain

$$
\begin{aligned}
\left(S_{20,0.1}\right)^{\prime}(0.9510)= & 0.1 \times 20(1-2 \times 0.9510)+2 \pi \times 0.1 \times 0.2 \sin (4 \pi \times 0.9510) \\
& +(1-0.1)=-0.9766 \text { radian }
\end{aligned}
$$

Now, substituting this value in (3.8), we have

$$
\sigma=\ln |-0.9766|=-0.0103 \text {. }
$$

Thus, the estimated value of the Lyapunov exponent is -0.0103 , i.e., $\sigma<$ 0 , therefore, the fixed point 0.9510 becomes stable attractor for the map $S_{20,0.1}(0.9510)$.

We have interpreted this fact experimentally in Fig. 17. We notice that $\sigma<0$ for $0<\mu \leq 20.34$, therefore, the system exhibits stable and fixed orbit for this extended range of $\mu$. After this range of $\mu$, system starts to show unstable behavior.

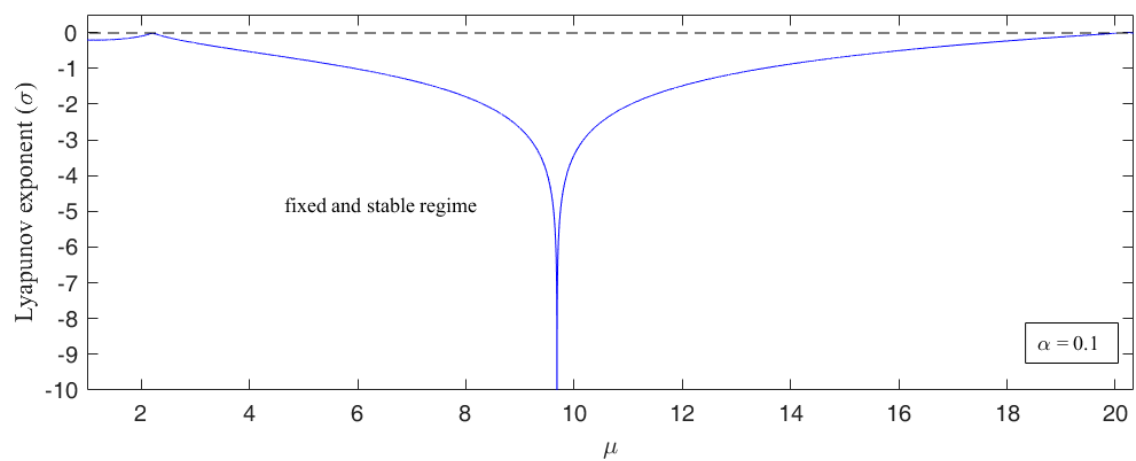

Fig. 17. Lyapunov exponent plot of SLSSM for $x_{0}=0.5,1 \leq \mu \leq 20.34$ at $\alpha=0.1$

\subsection{A new experimental analysis of SLSSM through combined study of bifurcation and Lyapunov exponent}

In this section, we try to understand the complex behavior of proposed system SLSSM by combining two diagrams, i.e., say, bifurcation diagram and 
Lyapunov exponent diagram. This experimental approach enables us to examine the accurate value of parameter $\mu$ obtained in previous sections at which system alters its behavior. We have divided the whole region of the dynamical system SLSSM into different regimes separated by a magenta dotted line as shown in figures. Fig. 18 shows the combined graphical representation of bifurcation and Lyapunov exponent for $1 \leq \mu \leq 4.5$ at $\alpha=0.9$. The system possesses two regimes, stable periodic regime and chaotic regime, separated by a magenta dotted line at $\mu=3.86$, which is the maximum value of $\mu$ for the system to be stable, thereafter chaos occurs. At $\mu=3.86$, Lyapunov exponent $\sigma$ is negative. We divide the whole region of SLSSM into three regimes (stable, undefined and chaotic regime) at specific values of parameter $\mu$ as illustrated in Figs. 19 and 20 for $\alpha=0.5$ and $\alpha=0.1$ respectively. From the figures, it can be noticed that system maintains its stability for a larger value of $\mu$ by decreasing the value of $\alpha$. Moreover, when $\sigma$ exceeds the value 0 , the system intends to be chaotic.

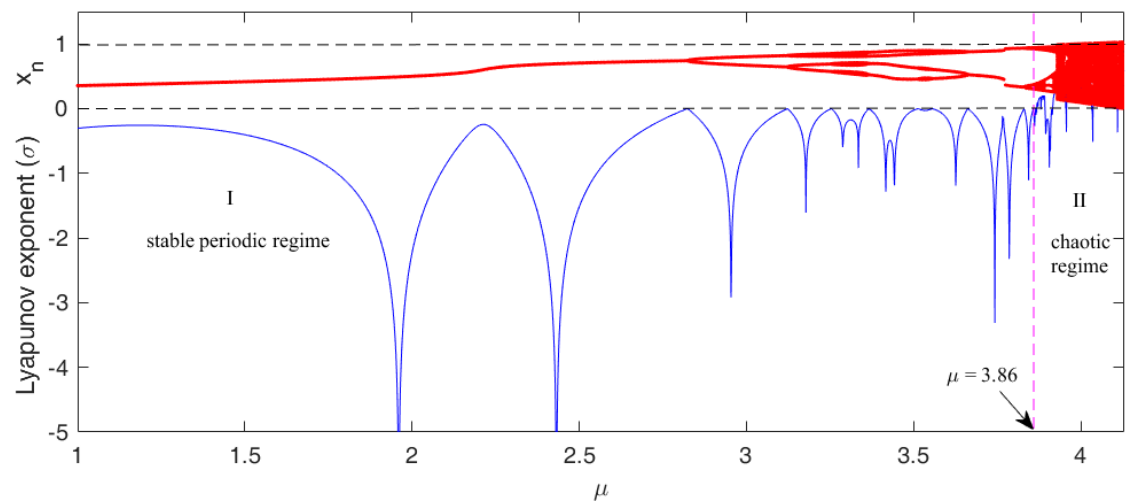

Fig. 18. Bifurcation plot versus Lyapunov exponent diagram of SLSSM for $1 \leq \mu \leq 4.5$ at $\alpha=0.9$ 


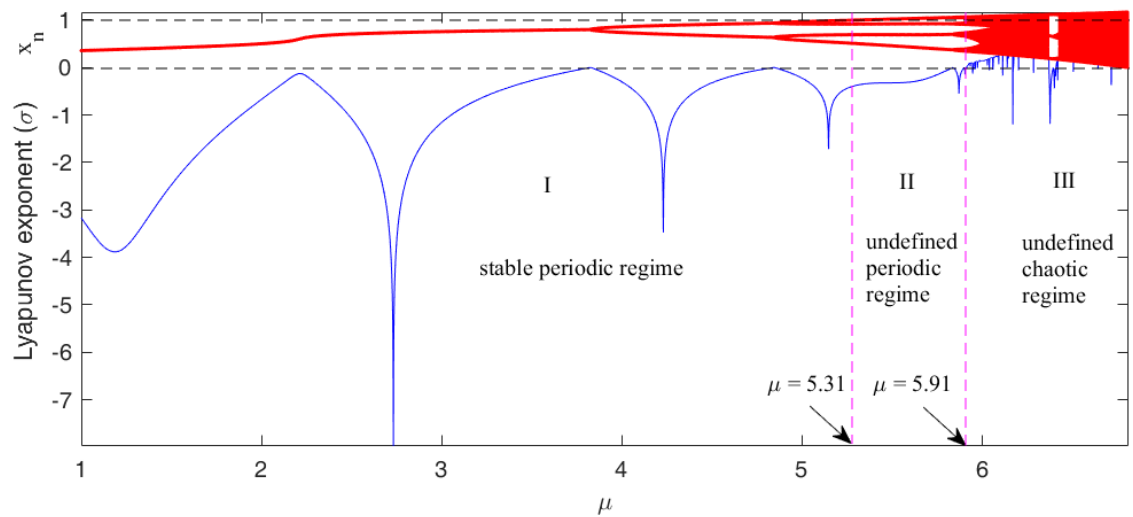

Fig. 19. Bifurcation plot versus Lyapunov exponent diagram of SLSSM for $1 \leq \mu \leq 7$ at $\alpha=0.5$

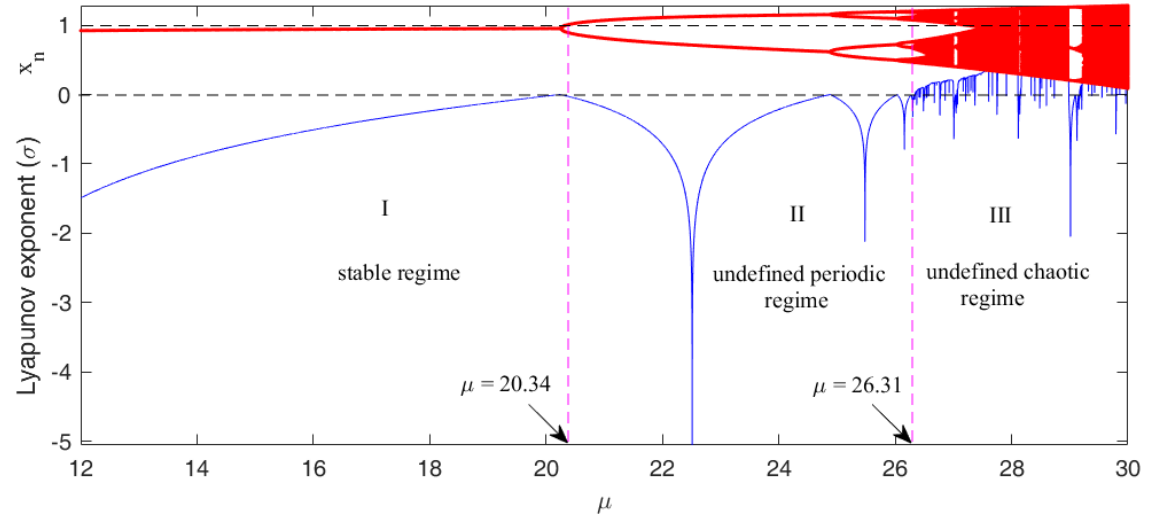

Fig. 20. Bifurcation plot versus Lyapunov exponent diagram of SLSSM for $12 \leq \mu \leq 30$ at $\alpha=0.1$

\section{Superiority of SLSSM}

To prove the excellence and superiority of SLSSM, we compare its stability and chaos performance with existing one dimensional maps in terms of bifurcation plots.

\subsection{Stability performance of SLSSM}

In order to facilitate comparison, we compare the stability performance of SLSSM with existing one dimensional maps: logistic map given by (1.1), sine 
map represented by the relation (1.2) and squared sine logistic map [36].

From Fig. 21, we observe that SLSSM remains stable for $0<\mu \leq 20.34$ as we have already shown in Subsection 3.3. Sine map is stable for $0<$ $\lambda \leq 0.86$ while logistic map shows its stable behavior for $0<\mu \leq 3.57$ as shown by Subfigures 21a and 21b respectively. Also, one dimensional squared sine logistic map attains its stability performance for $0<\mu \leq 3.5$. Hence, SLSSM has largest range of stability which is very higher than the existing one dimensional chaotic maps.

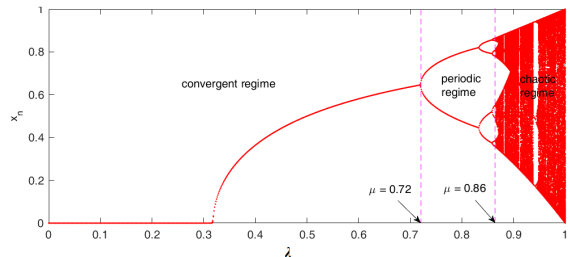

(a)

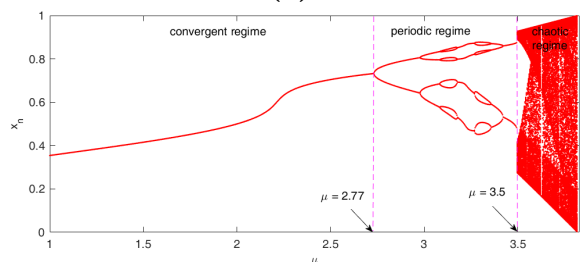

(c)

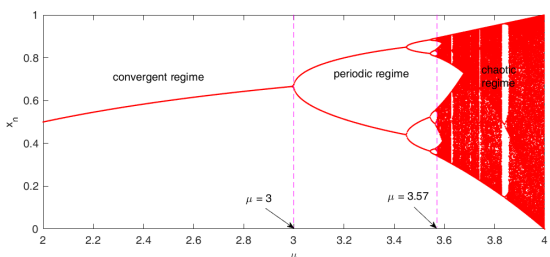

(b)

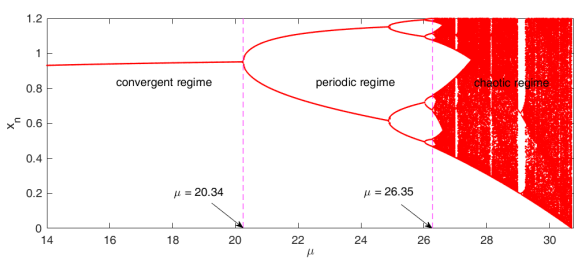

(d)

Fig. 21. Bifurcation plots (a) sine map (b) logistic map (c) squared sine logistic map and (d) standard logistic sine square map (SLSSM).

\subsection{Chaos performance of SLSSM}

The chaos performance of a chaotic map is very significant tool for image encryption. The properties of chaotic maps such as: extreme sensitivity on initial conditions, ergodicity and unpredictable behavior are very suitable for image encryption. In 1997, the chaos based image encryption was firstly introduced by Fridrich [42]. Subsequently, authors [37, 38] developed new chaotic system by combining two different chaotic systems which has better chaos performance to design a higher security encryption structure. Motivated by them, here, we also evaluate the chaos performance of SLSSM so that it can be used in image encryption.

In Fig. 22, we evaluate the chaos performance of sine map, logistic map 
and SLSSM by magnifying the chaotic regimes of their respective bifurcation diagrams represented in Fig. 21. From the figure, we notice that SLSSM shows chaotic behavior for $26.6 \leq \mu \leq 30.8$. Sine map has chaotic behavior for $0.87 \leq \lambda \leq 1$. The logistic map remains chaotic for $3.58 \leq \mu \leq 4$. Therefore, the SLSSM has a wider chaotic range and therefore, more complex chaotic behavior than the other existing one dimensional chaotic maps.

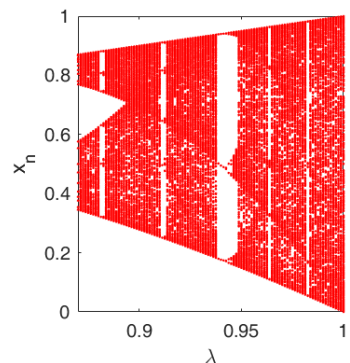

(a)

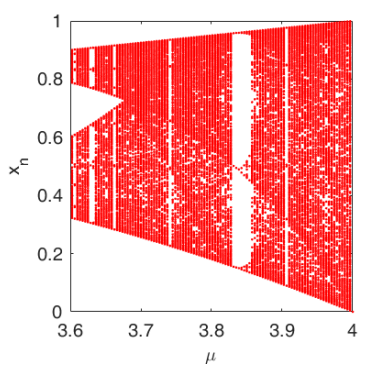

(b)

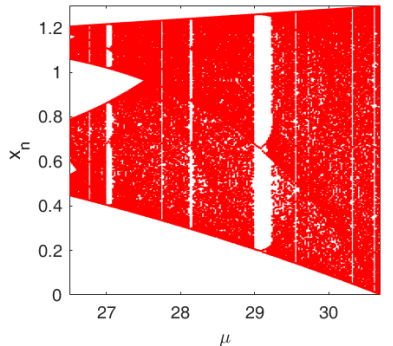

(c)

Fig. 22. Chaos performance (a) sine map (b) logistic map (C) standard logistic sine square map (SLSSM).

Remark 4.1. The stability and chaos performance of SLSSM is wider than that of sine map and logistic map. Therefore, this map might be applied in image encryption to obtain better results than existing one dimensional maps.

\section{Discussion and Conclusion}

We propose a new dynamical system Standard Logistic Sine Square Map (SLSSM) by combining logistic map and sine square map. The new system SLSSM possesses two extra control parameters which alters the whole dynamics of the system as compared to logistic map and sine map. The control parameters are denoted by $\alpha, \mu$ and $\lambda$. The entire dynamical behavior of SLSSM is analyzed adopting the fixed point and stability analysis, time series representation, bifurcation diagram, Lyapunov exponent and combined diagrams of bifurcation and Lyapunov exponent. The following concluding remarks are drawn from our investigation:

1. The fixed point analysis technique has been utilized to calculate the fixed points of the proposed system (3.1). Further, the stability performance of the uncontrolled system has also been checked. The range of convergence and stability of SLSSM can be increased upto $\mu=16.75$ by controlling parameters (see Table 1). 
2. The complicated and rich periodic dynamical behavior of SLSSM has been experimentally presented using time series representation at $\alpha=$ $0.9,0.5,0.1$ to verify the stability results obtained by using fixed point analysis.

3. The bifurcation analysis is used to evaluate various dynamic properties of SLSSM such as fixed point, period doubling, reduction in periodicity and route from periodic to chaos for $0<\mu \leq 4.5$ at $\alpha=0.9$.

4. The irregularity of the dynamical system has been illustrated numerically and experimentally by Lyapunov exponent technique. Further, combined diagrams of bifurcation and Lyapunov exponent are demonstrated to determine various regimes of the system SLSSM.

5. This worthwhile study has been carried out to analyze and control the dynamic complication of proposed SLSSM. It is observed that by choosing control parameters carefully, the dynamics of SLSSM can be effectively controlled.

6. Also, SLSSM has wider range of stability and chaos than that of sine map, logistic map and squared sine logistic map (see, Section 4). Therefore, our system might be used in all nonlinear phenomenons such as cryptography, chaotic noise analog generator, control traffic and traffic signals etc. where one dimensional dynamical systems (logistic map, sine map etc.) have been used so far.

7. Our future work is to study interesting problems concerning control and synchronization of new system (SLSSM). Also, we will apply the chaotic map SLSSM in image encryption to obtain better results.

\section{References}

[1] A. Hastings, C.L. Hom, S. Ellner, P. Turchin, H.C.J. Godfray, Chaos in Ecology: Is Mother Nature a Strange Attractor?, Annu. Rev. Ecol. Syst. 24 (1993), 1-33.

[2] D. Rickles, P. Hawe, A. Shiell, A Simple Guide to Chaos and Complexity, J. Epidemiol. Commun. Health. 61 (2007), 933-937.

[3] M. Berezowski, M. Lawnik, Identification of fast-changing signals by means of adaptive chaotic transformations, Nonlinear Anal. Model. Control, 19 (2014), 172-177. 
[4] M. Lawnik, M. Berezowski, Identification of the oscillation period of chemical reactors by chaotic sampling of the conversion degree, Chem. Process Eng. 35 (2014), 387-393.

[5] M. Lawnik, Generation of numbers with the distribution close to uniform with the use of chaotic maps, Proceedings of the 4th International Conference on Simulation and Modeling Methodologies, Technologies and Applications, (2014), 451-455.

[6] S. Kumari, R. Chugh, J. Cao, C. Huang, On the construction, properties and Hausdorff dimension of random Cantor one pth set, AIMS Mathematics, 5 (2020), 3138-3155.

[7] S. Kumari, R. Chugh, J. Cao, C. Huang, Multi Fractals of Generalized Multivalued Iterated Function Systems in b-Metric Spaces with Applications, Mathematics 7 (2019), 967.

[8] S. Kumari, R. Chugh, Novel fractals of Hutchinson Barnsley operator in Hausdorff $g$-metric spaces, Poincare Journal of Analysis \& Applications, 7 (2020), 99-117.

[9] S. Kumari, M. Kumari, R. Chugh, Dynamics of superior fractals via Jungck SP orbit with $s$-convexity, Annals of the University of CraiovaMathematics and Computer Science Series, 46(2), 2019, 344-365.

[10] S. Kumari, M. Kumari, R. Chugh, Graphics for complex polynomials in Jungck-SP orbit, IAENG International Journal of Applied Mathematics, 49 (2019), 568-576.

[11] R.L. Devaney, An Introduction to Chaotic Dynamical Systems, 2nd ed., Westview Press, USA 2003.

[12] K.T. Alligood, T.D. Sauer, J.A. Yorke, Chaos : An Introduction to Dynamical Systems, Springer, New York 1996.

[13] R.A. Holmgren, A first course in discrete dynamical systems, SpringerVerlag; 1994.

[14] R. May, Simple mathematical models with very complicated dynamics, Nature, 261 (1976), 459-475.

[15] S. Kumar, M. Kumar, R. Budhiraja, M.K. Das, S. Singh, A secured cryptographic model using intertwining logistic map, Procedia Computer Science, 143 (2018), 804-811. 
[16] C. Han, An image encryption algorithm based on modified logistic chaotic map, Optik, 181 (2019), 779-785.

[17] Z. Hua, Y. Zhou, Image encryption using 2D Logistic-adjusted-Sine map, Inf. Sci. 339 (2016), 237-253.

[18] L.P.L. de Oliveira, M. Sobottka, Cryptography with chaotic mixing, Chaos, Solitons \& Fractals, 3 (2008), 466-471.

[19] P. Shang, X. Li, S. Kame, Chaotic analysis of traffic time series, Chaos, Solitons \& Fractals, 25 (2005), 121-128.

[20] S.C. Lo, H.J. Cho, Chaos and control of discrete dynamic traffic model, J. Franklin Inst. 342 (2005), 839-851.

[21] M. McCartney, A discrete time car following model and the bi-parameter logistic map, Commun. Nonlinear Sci. Numer. Simul. 14 (2009), 233-243.

[22] Ashish, J. Cao, R. Chugh, Chaotic behavior of logistic map in superior orbit and an improved chaos-based traffic control model, Nonlinear Dynamics, 2018, 1-17. https://doi.org/10.1007/s11071-018-4403-y.

[23] T. Nagatani, Vehicular motion through a sequence of traffic lights controlled by logistic map, Physics Letters A, 372 (2008), 5887-5890.

[24] T. Nagatani, N. Sugiyama, Vehicular traffic flow through a series of signals with cycle time generated by a logistic map, Physica A, 392 (2013), 851856.

[25] S. Kumari, R. Chugh, A novel four-step feedback procedure for rapid control of chaotic behavior of the logistic map and unstable traffic on the road, Chaos, 30 (2020), 123115.

[26] N. Singh, A. Sinha, Chaos-based secure communication system using logistic map, Opt. Lasers Eng. 48 (2010), 398-404.

[27] J.S. Martin, M.A. Porter, Convergence time towards periodic orbits in discrete dynamical systems, PLOS One, 9 (2014), 1-9.

[28] R.V. Medina, A.D. Mendez, J.L. Rio-Correa, J.L. Hernandez, Design of chaotic analog noise generators with logistic map and MOS QT circuits, Chaos, Solitons \& Fractals, 40 (2009), 1779-1793.

[29] R. Chugh, A. Kumar, S. Kumari, A novel epidemic model to analyze and control the chaotic behavior of covid-19 outbreak, Bulletin of the Transilvania University of Brasov, Series III: Mathematics, Informatics, Physics, 13(2020), 479-508. 
[30] F.G. Xie, B.L. Hao, ymbolic Dynamics of the Sine-square Map, Chaos, Solitons \& Fractals, 3 (1993), 47-60.

[31] P. Philominathan, P. Neelamegam, S. Rajasekar, Statistical dynamics of sine-square map, Physica A, 242 (1997), 391-408.

[32] B. Saha, S.T. Malasani, J.B. Seventline, Application of Modified Chaotic Sine Map in Secure Communication, Int. J. Comput. Appl. 113 (2015), $9-14$.

[33] H. Ogras, M. Turk, A Secure Chaos-based Image Cryptosystem with an Improved Sine Key Generator, American Journal of Signal Processing, 6 (2016), 67-76.

[34] X. Jie1, C. Pascal, F.P. Daniele, T.A. Kaddous, L. KePing, Chaos generator for secure transmission using a sine map and an RLC series circuit, Science in China Series F: Information Sciences, 53 (2010), 129-136.

[35] G.C. Wu, D. Baleanu, S.D. Zeng, Discrete chaos in fractional sine and standard maps, Physics Letters A, 378 (2014), 484-487.

[36] Egydio de Carvalho R., Edson D. Leonel: Squared sine logistic map, Physica A, 463 (2016), 37-44.

[37] J. Wu, X. Liao, B. Yang, Image Encryption Using 2D Henon-Sine Map and DNA Approach, Signal Process. 153 (2018), 11-23, doi: 10.1016/j.sigpro.2018.06.008.

[38] Z. Hua, F. Jin, B. Xu, H. Huang, 2D Logistic-Sine-Coupling Map for Image Encryption, Signal Process. 149 (2018), 148-161, doi: 10.1016/j.sigpro.2018.03.010.

[39] W.R. Mann, Mean value methods in iteration, Proc. Am. Math. Soc. 4 (1953), 506-510 .

[40] M. Rani, R. Agarwal, A new experimental approach to study the stability of logistic map, Chaos, Solitons \& Fractals, 41 (2009), 2062-2066.

[41] Ashish, J. Cao, A Novel Fixed Point Feedback Approach Studying the Dynamical Behaviors of Standard Logistic Map, Internat. J. Bifur. Chaos 29(2019), 1950010 (16 pages).

[42] J. Fridrich, Image encryption based on chaotic maps, in Proceedings of IEEE International Conference on Systems, Man and Cybernetics(ICSMC`97), 2(1997), 1105-1110. 
ON THE COMPLEX AND CHAOTIC DYNAMICS OF STANDARD LOGISTIC SINE SQUARE MAP

Sudesh Kumari,

Department of Mathematics,

Government College for Girls Sector-14,

Gurugram 122001, India.

Email: tanwarsudesh10@gmail.com

Renu Chugh,

Department of Mathematics,

Maharshi Dayanand University,

Rohtak-124001, India.

Email: chugh.r1@gmail.com

Radu Miculescu,

Faculty of Mathematics and Computer Science,

Transilvania University of Brasov,

Brasov 500091, Romania.

Email: radu.miculescu@unitbv.ro 